EPJ Web of Conferences 70, 00033 (2014)

DOI: $10.1051 /$ epjconf/ 20147000033

(C) Owned by the authors, published by EDP Sciences, 2014

\title{
The Old New Frontier: Studying the CERN-SPS Energy Range with NA61/SHINE
}

\author{
Marek Szuba ${ }^{1, a}$ for the NA61/SHINE Collaboration ${ }^{2}$ \\ ${ }^{1}$ Karlsruhe Institute of Technology, Germany \\ ${ }^{2}$ CERN, Geneva, Switzerland
}

\begin{abstract}
With the Large Hadron Collider entering its third year of granting us insight into the highest collision energies to date, one should nevertheless keep in mind the unexplored physics potential of lower energies. A prime example here is the NA61/SHINE experiment at the CERN Super Proton Synchrotron. Using its large-acceptance hadronic spectrometer, SHINE aims to accomplish a number of physics goals: measuring spectra of identified hadrons in hadron-nucleus collisions to provide reference for accelerator neutrino experiments and cosmic-ray observatories, investigating particle properties in the large transverse-momentum range for hadron+hadron and hadron+nucleus collisions for studying the nuclear modification factor at SPS energies, and measuring hadronic observables in a particularly interesting region of the phase diagram of strongly-interacting matter to study the onset of deconfinement and search for the critical point of stronglyinteracting matter with nucleus-nucleus collisions. This contribution shall summarise results obtained so far by NA61/SHINE, as well as present the current status and plans of its experimental programme.
\end{abstract}

\section{Introduction}

There is no doubt that studies of particle collisions at the highest available energies can and do result in important scientific results; the string of discoveries from the Tevatron at Fermilab and the recent observation at the Large Hadron Collider at CERN of what is likely the Higgs boson attest to that. Even so, one should keep in mind the unexplored physics potential of collisions at lower energies. Data from experiments such as those at the Super Proton Synchrotron (SPS) at CERN, operating well below the high-energy frontier, continue to contribute to expanding our knowledge of matter, our world and the universe. Examples of projects depending on such data include:

\subsection{Measurement of Neutrino Oscillation}

Predicted by Pontecorvo 1957 and since then observed experimentally, neutrino oscillation is a quantum-mechanical phenomenon which can make a neutrino produced with a specific flavour be observed later as possessing different flavour [1]. It stems from differences in quantum-phase propagation of different neutrino mass eigenstates and as such requires neutrinos to have mass. One way

\footnotetext{
a e-mail: Marek.Szuba@kit.edu
} 
to obtain accurate measurements of neutrino oscillation is to perform it on neutrinos produced in a controlled environment of a particle accelerator: a high-energy beam of protons is collided against a graphite target to produce positive pions and kaons, which subsequently decay into muons and muon neutrinos.

One experiment measuring oscillation of beam neutrinos is T2K in Japan, directing particles from the J-PARC facility in Tokai towards the Super-Kamiokande detector $295 \mathrm{~km}$ away in Kamioka [2]. Physics goals of T2K are to obtain one of the first measurements of the $\theta_{13}$ mixing angle of the Pontecorvo-Maki-Nakagawa-Sakata (PMNS) neutrino-mixing matrix, to improve precision of measurements of the $\theta_{23}$ mixing angle and the mass difference $\Delta m_{23}^{2}$, and in the future to search for $v \mathrm{CP}$ violation. Other experiments of this sort include MINOS and NOvA (NuMI beam at Fermilab), LBNE (upcoming new $v$ beam at Fermilab), and OPERA and ICARUS (CNGS beam at CERN) [3][4][5][6][7].

Unfortunately even with well-defined primary beams, estimating the resulting neutrino flux is not a trivial matter. In order to achieve adequate beam intensity the graphite target must be long, leading to secondary interactions. Moreover, a non-negligible contribution to the flux is made by interactions with support structures such as the target holder and cooling systems. In short, good knowledge of properties of the resulting neutrino beam requires good knowledge of hadron production in the target - which in turn may be much easier to study using an identical target at a fixed-target facility oriented towards hadron spectroscopy than having to set up necessary detectors in situ. Given the energies of proton beams typically used for production of neutrino beams, the CERN SPS complex (indeed, in case of the CNGS beam the proton accelerator in question is the SPS) is a perfect candidate for this task.

\subsection{Hadron Production in Extensive Air Showers}

Another domain which can benefit from hadroproduction measurements in the energy range of the SPS are studies of extensive air showers (EAS) produced in Earth's atmosphere by cosmic rays. Detecting and measuring EAS is the standard technique for studying ultra high-energy cosmic rays, as such particles reach our planet so infrequently and from so many different directions that it is virtually impossible to gather a statistically significant sample through direct measurements (i.e. using satellite- or balloon-based detectors to observe cosmic-ray particles themselves); using EAS detectors makes it both technically and financially easier to cover a much wider area. Examples of modern EAS experiments include KASCADE-Grande in Germany, Pierre Auger Observatory in Argentina and the Telescope Array in the United States [8][9][1].

Improved observation rates of EAS-based cosmic-ray experiments come at a cost of having to accurately reconstruct properties of the original particle from what has undergone multiple levels of interactions with the atmosphere before reaching the detectors. In particular, evolution of the hadronic component of showers remains relatively poorly understood. With primary energies of ultra high-energy cosmic rays remaining largely out of range of man-made accelerators, one is required to employ simulations to provide reference for such evolution — which in turn requires careful tuning of models used for this purpose.

This is where measuring hadron spectra at the SPS comes in. Although its energy range falls several orders of magnitude short of the energy of cosmic rays observed by EAS detectors $\left(E_{0}=10^{15}-\right.$ $10^{20} \mathrm{eV}$, it provides an excellent match to the energy of the last generation of hadronic interactions in the shower. As many experimental observables (e.g. the number of muons detected by surface detectors) are directly tied to this stage of shower evolution, studying it at the SPS is expected to yield significantly improved predictions. 


\subsection{High- $p_{T}$ Hadrons in $\mathrm{p}+\mathrm{p}$ and $\mathrm{p}+\mathrm{A}$ Colliisions}

Proton-proton and proton-nucleus collisions constitute important reference systems for a wide range of different studies (for instance spectra, scaling with the number of wounded nucleons or binary collisions, nuclear modification factor and Cronin effect, among others) in nucleus-nucleus reactions. However, in the past data sets of this sort at SPS energies and below were relatively small. This problem becomes particularly pressing in case of high- $p_{T}$ hadrons, originating from hard scatterings and thus useful for probing the perturbative-quantum chromodynamics (QCD) regime of strong interactions - they are produced so rarely that most such elementary collisions contain none at all, further reducing effective sample size. Fortunately, development of sophisticated detectors and readout components capable of coping with high event rates of high-luminosity machines such as the LHC has made it possible to also improve event rates of lower-energy detectors, allowing at last collection of large-statistics $\mathrm{p}+\mathrm{p}$ and $\mathrm{p}+\mathrm{A}$ data sets. Analysis of this data is expected to greatly improve precision of many existing results as well as allow new, hitherto-infeasible studies.

\subsection{Critical Point and the Onset of Deconfinement of QCD Matter}

Theoretical predictions based on QCD tell us that above certain energy density, quarks and gluons previously confined to hadrons can undergo a phase transition into the quark-gluon plasma - a state in which they can be considered free. The energy range of the SPS is very important for studying this transition because at these energies one can produce collisions both right below and right above the energy threshold for deconfinement [1]. Moreover, it is also in this energy range that we now expect the critical point of the QCD phase diagram [1].

A number of experimental observables were proposed to be sensitive to the onset of deconfinement; these include changes in energy dependence of the pion yield per wounded nucleon ("the kink"), of the $\frac{\left\langle K^{+}\right\rangle}{\left\langle\pi^{+}\right\rangle}$ratio ("the horn") and of the mean transverse mass ("the step") [1]. On the other hand, the emergence of the critical point is predicted to be observable in event-by-event fluctuations of e.g. multiplicity and average transverse momentum [1]. Indications in favour of this hypothesis has been observed both at the SPS and elsewhere [1][1][1].

\section{The NA61/SHINE Experiment}

\subsection{Overview and Physics Goals}

NA61/SHINE (SPS Heavy Ion and Nutrino Experiment) is a fixed-target experiment located in the North Area of the CERN SPS, using a large-acceptance hadronic spectrometer to study a wide range of phenomena in a number of different hadron-hadron, hadron-nucleus and nucleus-nucleus reactions [1]. It is the successor of the NA49 experiment, which took data in the years 1994-2002, and reuses most of its predecessor's hardware and software [1]. Its large acceptance (around $50 \%$ for $\left.p_{T} \leq 2.5 \mathrm{GeV} / \mathrm{c}\right)$, high momentum resolution $\left(\sigma(p) / p^{2} \approx 10^{-4}(\mathrm{GeV} / \mathrm{c})^{-1}\right)$ and tracking efficiency (over $95 \%$ ), and excellent particle-identification capabilities $\left(\sigma\left(\frac{\mathrm{d} E}{\mathrm{~d} x}\right) / \frac{\mathrm{d} E}{\mathrm{~d} x} \approx 4 \%, \sigma\left(t_{T o F}\right) \approx 100 \mathrm{ps}\right.$ ) make it an excellent tool for investigating hadron spectra.

NA61/SHINE has the following physics goals:

- search for the critical point on the phase diagram of strongly-interacting (QCD) matter and study in detail the onset of deconfinement by performing a two-dimensional scan of the phase diagram (see Figure 1),

- investigate production of high- $p_{T}$ hadrons in $\mathrm{p}+\mathrm{p}$ and $\mathrm{p}+\mathrm{Pb}$ collisions, 


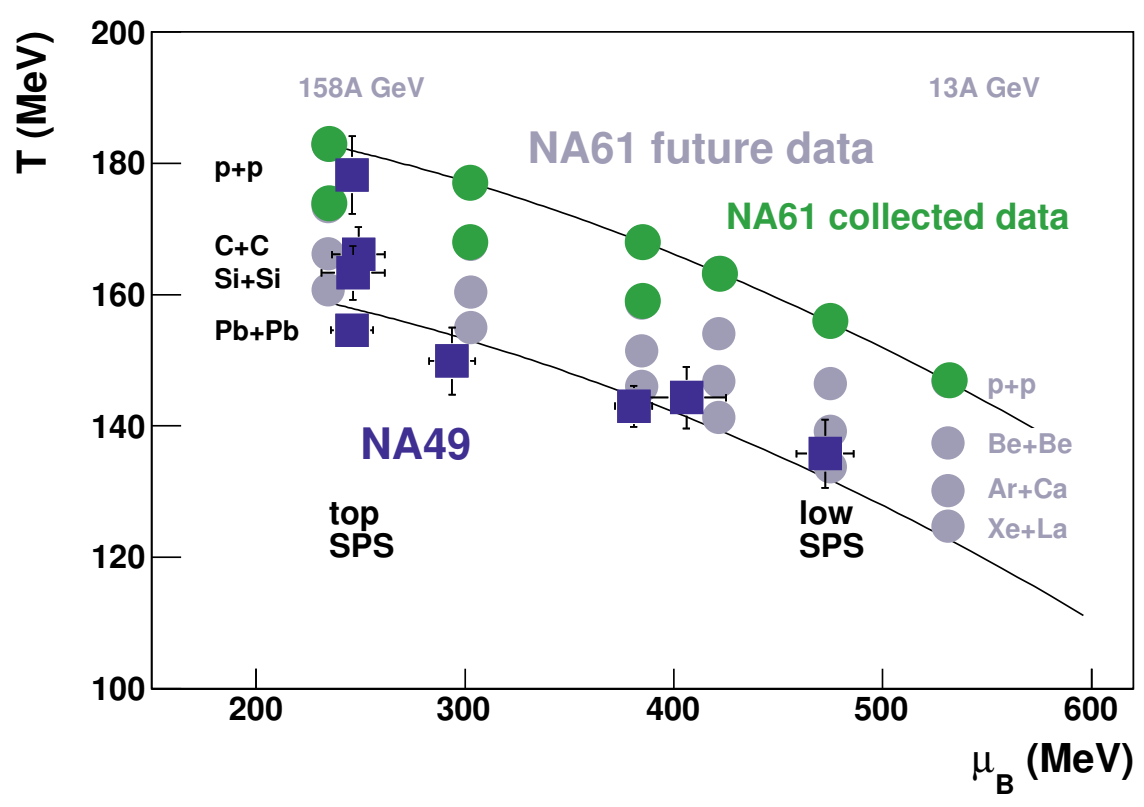

Figure 1. Estimated [1] (squares) and extrapolated (circles) chemical freeze-out points corresponding to NA49 and NA61/SHINE for both collected and future data.

- provide reference measurements for the T2K neutrino experiment by measuring hadron production in collisions of a proton beam at $31 \mathrm{GeV}$ with a carbon platelet and a replica of the T2 $\mathrm{K}$ target,

- provide reference measurements for current and future Fermilab neutrino experiments by measuring hadron production in collisions of a proton beam at 60,90 and $120 \mathrm{GeV}$ with a carbon platelet and a replica of the NuMI target,

- perform reference measurements of hadron production in $\mathrm{p}+\mathrm{p}, \mathrm{p}+\mathrm{C}$ and $\pi+\mathrm{C}$ interactions, for cosmic-ray extended air-shower experiments: KASCADE, KASCADE-Grande and Pierre Auger Observatory.

\subsection{Detector Set-up}

Figure 2 shows a diagram of the NA61/SHINE apparatus. The primary, tracking detectors are four large-volume Time Projection Chambers (TPCs) inherited from NA49. Two of them (Vertex TPCs) are located inside superconducting magnets along the beam line, the other two (Main TPCs) are placed symmetrically to the beam line further downstream. Another, smaller chamber (Gap TPC) is placed between the VTPCs. Together, these chambers measure trajectories and momentum of particles as well as allow their identification through ionisation energy loss.

Just downstream of the MTPCs there exist three Time-of-Flight (ToF) walls, the side two inherited from NA49 and the central one added by NA61 in 2007. Measurements from these detectors complement particle-identification information in the momentum range where $\frac{\mathrm{d} E}{\mathrm{~d} x}$ alone is ambiguous. 


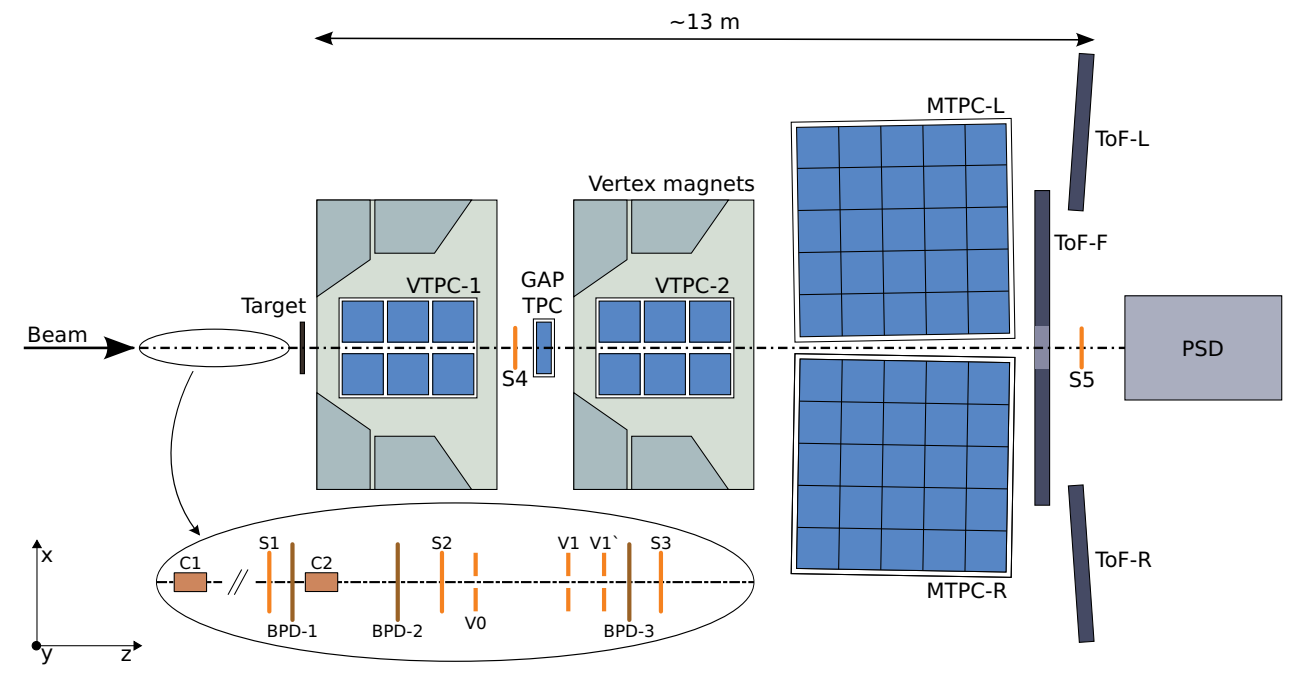

Figure 2. Layout of the NA61/SHINE experimental set-up (top view, not to scale). For details see [1].

The most downstream detector of the apparatus is the Projectile Spectator Detector (PSD), installed in 2011. The purpose of this calorimeter is to provide precise, high-granularity event-by-event measurements of the energy of non-interacting fragments of the projectile nuclei, making it possible to determine centrality of the collision and the orientation of the reaction plane.

Finally, a number of additional detectors installed in the beam line both upstream and downstream of the target monitor beam properties and provide trigger information.

It is also worth mentioning at this point that a sophisticated beam line set-up was designed and put in place by the CERN accelerators-and-beams department in collaboration with NA61 for the purpose of providing beryllium beams for SHINE. At the moment the only primary beams the SPS can provide are protons and lead ions, with argon and xenon to be made possible during the long shutdown of the accelerator complex in 2013-2014. As adding new species of primary beams is an immensely complicated task, beryllium beams for SHINE were instead produced in a fragmentationion beam line. Primary lead ions from the SPS were collided with a beryllium block far upstream in the beam line and the resulting fragments of the projectile were guided through a sequence of magnets, collimators, degraders and beam detectors in order to filter out beryllium ions. This configuration was proven to produce highly pure, stable beams of ${ }^{7} \mathrm{Be}$ over a wide range of $\mathrm{Pb}$-beam energies.

\subsection{Data Taking}

NA61/SHINE has been taking data since the year 2007 and so far has acquired over 150 million events in a variety of data sets. We focused primarily on data for cosmic-ray and neutrino physics in the first three years of running, and progressed to high- $p_{T}$ data and the exploration of the phase diagram in the following years. The presently-approved physics programme is foreseen to be concluded in the year 2015 . 
EPJ Web of Conferences

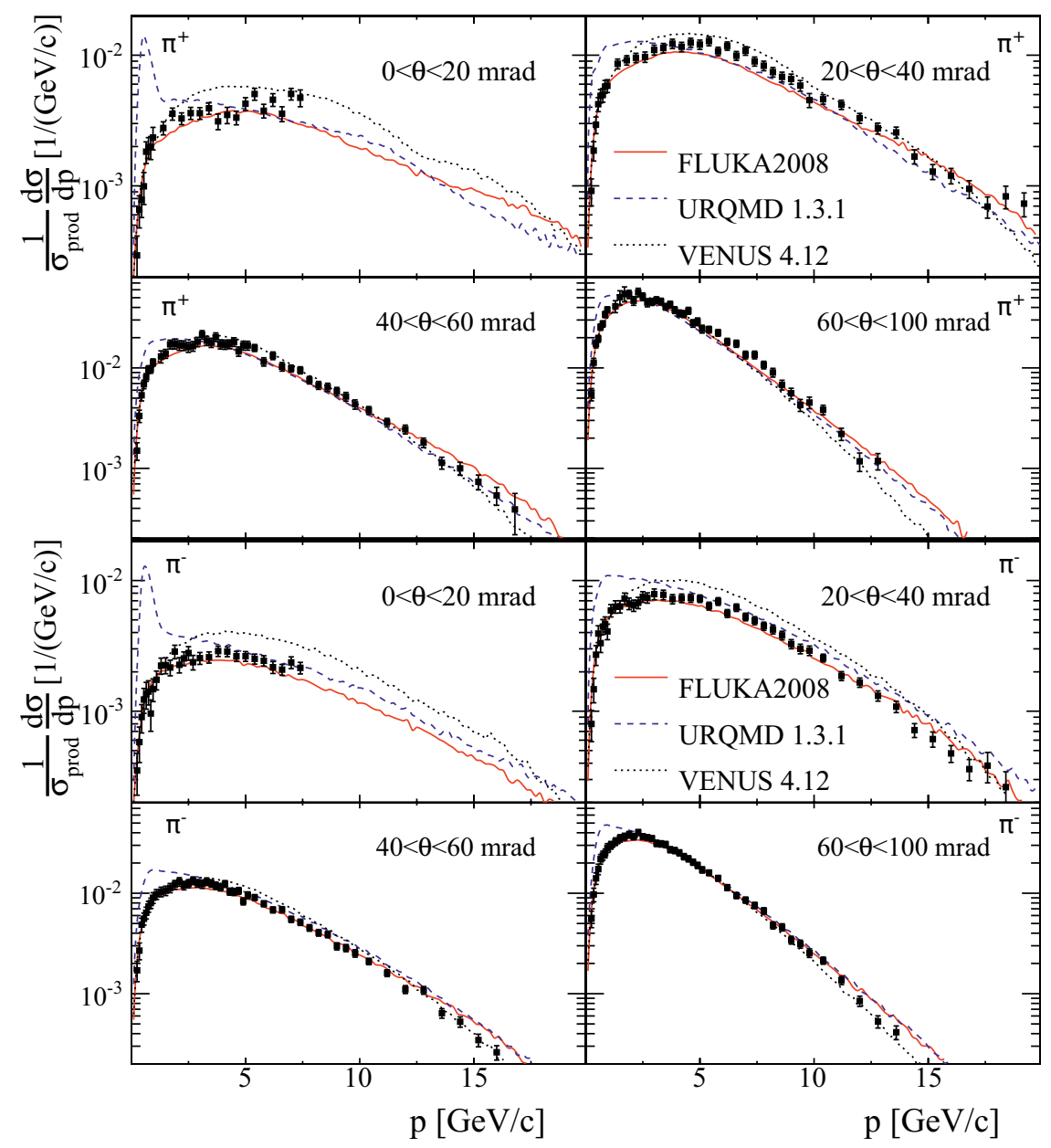

Figure 3. Inclusive spectra of positive (top) and negative (bottom) pions, in several polar-angle bins, measured by NA61/SHINE in $\mathrm{p}+\mathrm{C}$ collisions at $31 \mathrm{GeV}$ (points), compared to predictions (lines) from FLUKA 2008, UrQMD 1.3.1 and VENUS 4.12 [2].

\section{Results So Far}

The first $\pi^{ \pm}$(see Figure 3) and $K^{+}$(Figure 4) spectra from $\mathrm{p}+\mathrm{C}$ interactions at $31 \mathrm{GeV}$, based on data acquired in 2007, are published [2][2]. These results have already been used by the T2K experiment as reference for calculations of neutrino flux in the J-PARC beam, leading to their first measurement of the $\theta_{13} v$ mixing angle [2]. Moreover, comparison of pion spectra with models have made it possible to introduce improvements to UrQMD as well as to the implementation of FRITIOF in the Geant4 toolkit [2][2]. Results from data taken in 2009, offering a factor-of-ten increase in statistics as well improved particle identification through extension of the forward Time-of-Flight wall, are being prepared for release. 

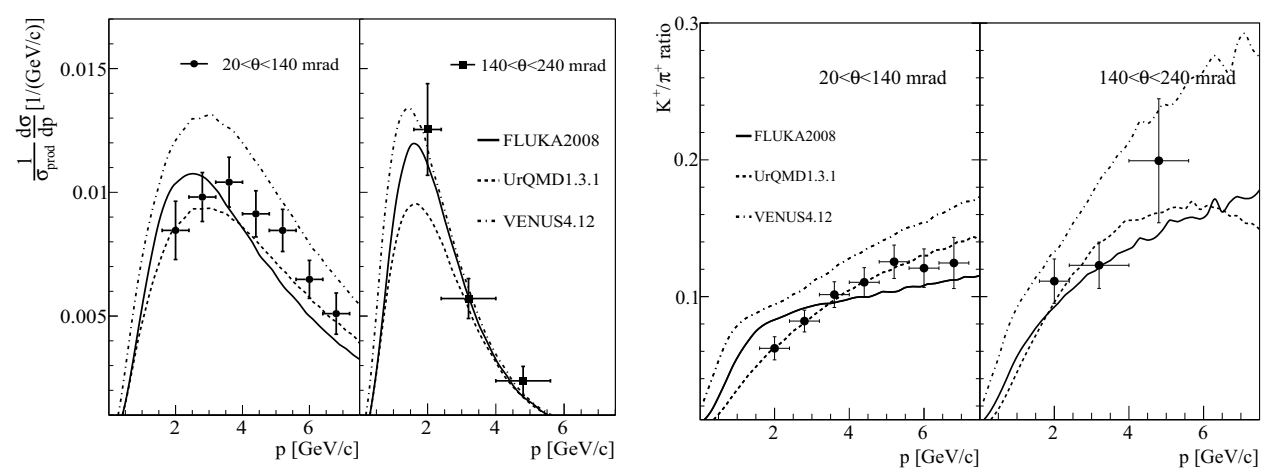

Figure 4. Left: Inclusive spectra of positively-charged kaons measured by NA61/SHINE in $\mathrm{p}+\mathrm{C}$ collisions at $31 \mathrm{GeV}$ (points), compared to predictions by various models (lines). Right: Comparison of the $K^{+} / \pi^{+}$ratio, using the above data along with results shown in Fig. 3, again compared to models [2].
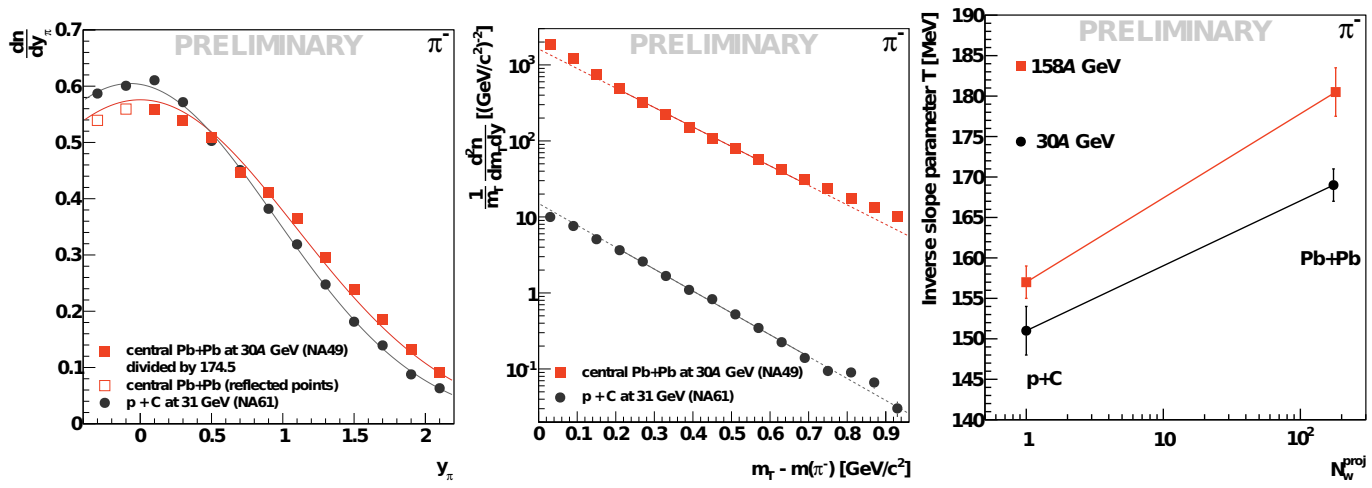

Figure 5. Left: Rapidity spectra of $\pi^{-}$in $\mathrm{p}+\mathrm{C}$ collisions at $31 \mathrm{GeV}$ and (scaled) central $\mathrm{Pb}+\mathrm{Pb}$ collisions at $30 \mathrm{~A} \mathrm{GeV}$, for $p_{T}<1 \mathrm{GeV} / \mathrm{c}$. Corresponding gaussian fits are also shown as lines. Middle: Transverse-mass spectra of $\pi^{-}$in the same reactions, for $0<y<0.2$. Dashed lines illustrate exponential fits to the data. Right: Inverse-slope parameters extracted from $m_{T}$ spectra for four different reactions measured by NA61 and NA49, with system size expressed as the number of wounded nucleons in the projectile.

Additional, preliminary results from $\mathrm{p}+\mathrm{C}$ interactions at $31 \mathrm{GeV}$ have been compared to measurements of $\mathrm{NA} 49$ in $\mathrm{Pb}+\mathrm{Pb}$ collisions [2]. Transverse-mass and forward rapidity spectra of negative pions were extracted and compared to NA49 results from central $\mathrm{Pb}+\mathrm{Pb}$ collisions at $30 \mathrm{~A} \mathrm{GeV}$ (see Figure 5). We have also begun investigating yields of $\Lambda, K_{S}^{0}$ and $\Delta^{++}$particles in these interactions.

Last but not least, we have recently presented the first preliminary $\pi^{ \pm}, K^{-}$and $p$ spectra from $\mathrm{p}+\mathrm{p}$ interactions at 40, 80 and $158 \mathrm{GeV}$ - reference data sets for the phase-diagram scan [2]; an overviews of these results can be found in Figure 6. Analogous measurements from $\mathrm{p}+\mathrm{p}$ events at 13, 20 and $31 \mathrm{GeV}$ are to follow suit. 

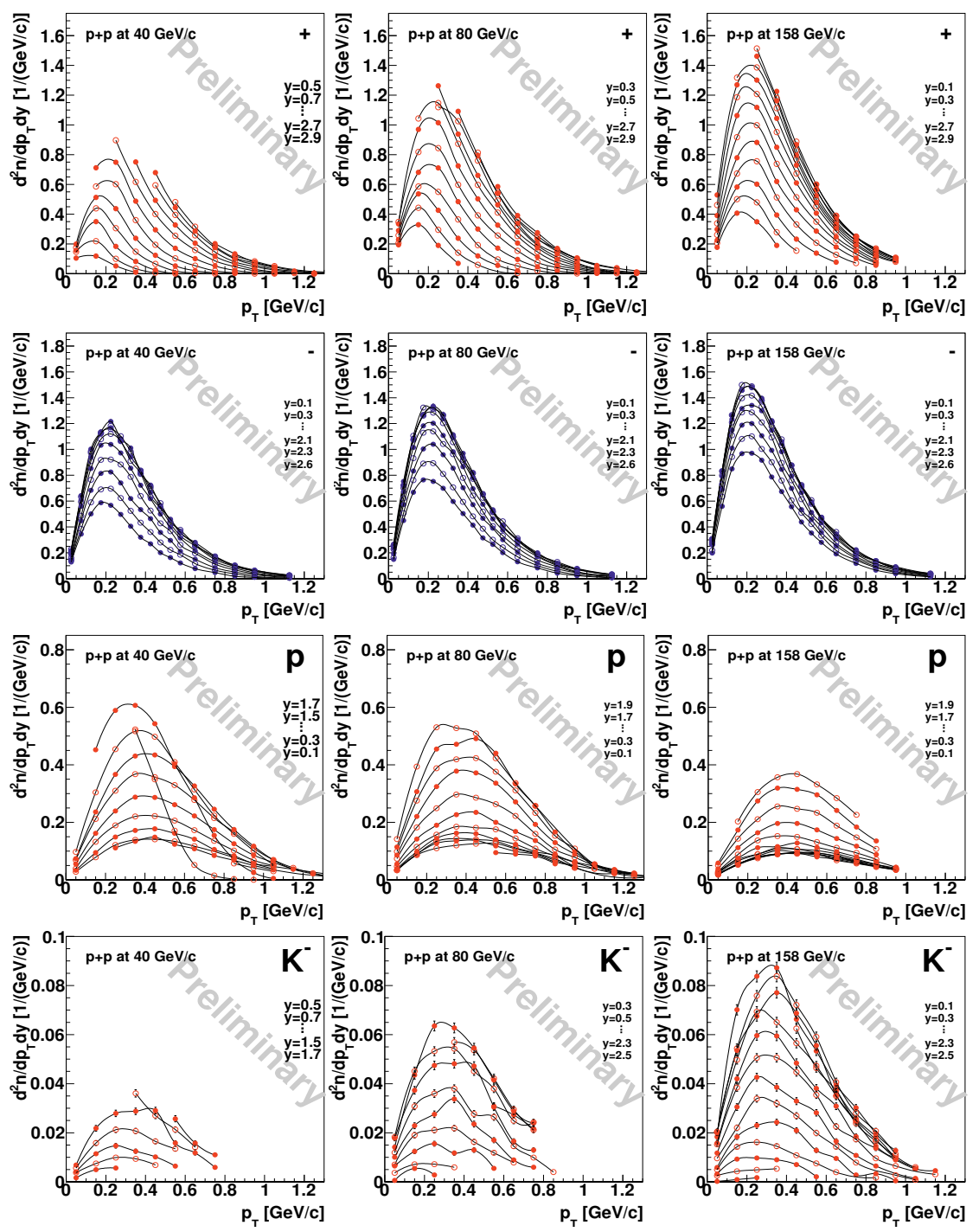

Figure 6. Double-differential spectra of $\pi^{+}, \pi^{-}, p$ and $K^{+}$(from top to bottom, respectively) in inelastic $\mathrm{p}+\mathrm{p}$ interactions at 40, 80 and $158 \mathrm{GeV}$. Only statistical uncertainties are shown; average systematic error is approximately $8 \%[2]$.

\section{Summary}

NA61/SHINE results have already provided important input for the calculation of the neutrino flux in the T2K beam which was used for the measurement of $\theta_{13}$. Moreover, models employed for simulating cosmic-ray showers were significantly constrained. The scan of the phase diagram of strongly interacting matter has begun and first results from $p+p$ collisions were presented. 


\section{References}

[1] U. Dore, D. Orestano, Rept.Prog.Phys. 71, 106201 (2008), arXiv:0811.1194 [hep-ex]

[2] Y. Itow et al. (T2K Collaboration), KEK-REPORT-2001-4, ICRR-REPORT-477-2001-7, TRIPP-01-05 (2001), arXiv: hep-ex/0106019

[3] E. Ables et al. (MINOS Collaboration), FERMILAB-PROPOSAL-0875, NUMI-L-79 (1995)

[4] D. Ayres et al. (NOvA Collaboration), FERMILAB-PROPOSAL-0929 (2004), arXiv:hep-ex/0503053

[5] M.C. Sanchez (LBNE DUSEL Collaboration), AIP Conf.Proc. 1222, 479 (2010)

[6] M. Guler et al. (OPERA Collaboration), CERN-SPSC-2000-028, CERN-SPSC-P-318, LNGSP25-00 (2000)

[7] F. Arneodo et al. (ICARUS collaboration), LNGS-P28-2001, ICARUS-TM-2001-03 (2001), arXiv: hep-ex/0103008

[8] A. Badea et al. (KASCADE-Grande Collaboration and the LOPES Collaboration), Nucl.Phys.Proc.Suppl. 136, 384 (2004), arXiv: astro-ph/0409319

[9] J. Abraham et al. (Pierre Auger Collaboration), Nucl.Instrum.Meth. A523, 50 (2004)

[10] K. Martens (Telescope Array Collaboration), Nucl.Phys.Proc.Suppl. 165, 33 (2007)

[11] C. Alt et al. (NA49 Collaboration), Phys.Rev. C77, 024903 (2008), arXiv:0710.0118 [nucl-ex]

[12] Z. Fodor, S. Katz, JHEP 0404, 050 (2004), arXiv: hep-lat/0402006

[13] M. Gazdzicki, M.I. Gorenstein, Acta Phys.Polon. B30, 2705 (1999), arXiv: hep-ph/9803462

[14] M.A. Stephanov, K. Rajagopal, E.V. Shuryak, Phys.Rev. D60, 114028 (1999), arXiv:hep-ph/9903292

[15] K. Grebieszkow (NA49 Collaboration), Nucl.Phys. A830, 547C (2009), arXiv:0907.4101 [nucl-ex]

[16] L. Kumar (STAR Collaboration), J.Phys.G G38, 124145 (2011), arXiv:1106.6071 [nucl-ex]

[17] N. Antoniou et al. (NA61/SHINE Collaboration), CERN-SPSC-2006-034, CERN-SPSC-P-330 (2006)

[18] S. Afanasev et al. (NA49 Collaboration), Nucl.Instrum.Meth. A430, 210 (1999)

[19] F. Becattini, J. Manninen, M. Gazdzicki, Phys.Rev. C73, 044905 (2006), arXiv:hep-ph/0511092

[20] N. Abgrall et al. (NA61/SHINE Collaboration), Phys.Rev. C84, 034604 (2011), arXiv: 1102.0983 [hep-ex]

[21] N. Abgrall et al. (NA61/SHINE Collaboration), Phys.Rev. C85, 035210 (2012), arXiv: 1112.0150 [hep-ex]

[22] K. Abe et al. (T2K Collaboration), Phys.Rev.Lett. 107, 041801 (2011), arXiv: 1106.2822 [hep-ex]

[23] V. Uzhinsky (2011), arXiv: 1107.0374 [hep-ph]

[24] V. Uzhinsky (2011), arXiv: 1109.6768 [hep-ph]

[25] K. Grebieszkow (NA61/SHINE Collaboration) (2012), arXiv: 1201.0742 [nucl-ex]

[26] S. Puławski (NA61/SHINE Collaboration), to be published in the proceedings of Quark Matter 2012 